\title{
Hafnium Oxide-containing Nanoparticles NBTXR3
}

National Cancer Institute

\section{Source}

National Cancer Institute. Hafnium Oxide-containing Nanoparticles NBTXR3. NCI

Thesaurus. Code C98280.

A suspension of nanoparticles containing inert inorganic hafnium oxide ( $\mathrm{HfO} 2)$ crystals with potential antineoplastic activity. Upon injection of NBTXR3 in the tumor, the hafnium oxide-containing nanoparticles accumulate into the tumor cells. Subsequent application of radiation beams to the tumor tissue causes $\mathrm{HfO} 2$ particles to emit huge amounts of electrons. This results in the formation of free radicals within the tumor cells, which in turn causes targeted destruction of the cancer cells. Compared to standard radiotherapy, because of the inert nature of NBTXR3, this agent emits electrons only during its exposure to radiation which improves radiotherapy efficiency. 